\title{
General relativistic plasmas around rotating black holes
}

\author{
Shinji Koide ${ }^{1}$ \\ ${ }^{1}$ Faculty of Science, Kumamoto University, 2-39-1 Kurokami, Kumamoto, 860-8555, Japan \\ email: koidesin@sci.kumamoto-u.ac.jp
}

\begin{abstract}
We show one of possible directions of general relativistic MHD (GRMHD) calculations of astrophysical relativistic jet formation. This was motivated by observations of radio knot ejections associated with high energy flares of an AGN in M87 and micro-quasars. We introduce a modified version of a solar flare model for the phenomenon. In the model, we have to consider a process beyond the ideal GRMHD. Especially, we focus on the special features of general relativistic plasmas around rotating black holes.
\end{abstract}

Keywords. black hole physics, gravitation, MHD, relativity, plasmas

\section{Introduction}

Numerical simulations of general relativistic MHD (GRMHD) have played important roles in investigation of plasma dynamics around rapidly rotating black holes (e.g., Koide, Shibata, and Kudoh 2006; McKinney 2006). For example, Koide et al. (2006) revealed that magnetic flux tubes bridging between an ergosphere and an accretion disk around a black hole, called "magnetic bridges", are unstable; they are twisted by the plasma in the ergosphere rapidly due to the frame-dragging effect, and are elongated by the magnetic pressure above the disk. During this process, the plasma is lifted outwardly with the expanding magnetic bridges to become a jet. McKinney (2006) performed a longer-term GRMHD simulation with an assumption of initial weak poloidal magnetic field in a disk around a rapidly rotating black hole. The numerical result showed the magnetic field is amplified due to the magnetorotational instability (MRI) and the $\Omega$ effect of magnetized plasma shear flow, and the amplified strong magnetic field is formed near the black hole to launch a jet. The jet is accelerated by the magnetic field over a long distance to become relativistic far from the black hole. It is believed that this simulation revealed the formation mechanism of a relativistic jet, while no corresponding analytic model is not clarified yet. We suppose that in the numerical simulation, the mechanism of magnetic bridges between the ergosphere and the disk played a main role for the launch of the jet. It is noted that zero resistivity (ideal MHD condition) was assumed in all of these GRMHD simulations. Within the framework of ideal GRMHD, the energy from the plasmas in the ergosphere is stored in the anti-parallel elongated magnetic flux tubes, and the energy is not released. In fact, the ideal GRMHD simulations show no explosive phenomena due to the energy release; they show gradual phenomena only in the longer term simulation (McKinney 2006). On the other hand, if we go beyond the ideal GRMHD, the magnetic energy of anti-parallel magnetic fields can be released suddenly by resistive phenomena.

Recent observations of M87 with X-ray/ $\gamma$-ray and radio showed the correlation of a high energy flare of nucleus of M87 and the radio knot ejection from the nucleus (Acciari et al. 2009). A similar correlation was also found in micro-QSOs more frequently (Mirabel and Rodrigus 2005). These explosive phenomena associated with knot ejection can not be explained within the ideal GRMHD. Similar correlation between a high energy flare 
and a knot ejection is found in a solar flare. The solar flare is explained by the magnetic reconnection model of elongated magnetic arcades (Shibata et al. 1995). The elongated magnetic flux tubes are formed by shear convection flow of the photosphere at the foot points of the magnetic flux tubes. In the flux tubes, a large amount of energy is stored because of very high magnetic Reynolds number and is released suddenly due to the magnetic reconnection with anomalous resistivity after the thin current sheet is formed. A similar model was considered to explain a flare and knot ejection of a proto-star. A non-relativistic MHD numerical simulation of interaction between plasma of a disk and corona around a proto-star and dipole magnetic field of the proto-star was performed by Hayashi, Shibata, and Matsumoto (1996). The simulation showed that magnetic bridges between the proto-star and the disk are twisted by the disk plasma rotating around the proto-star and the magnetic pressure increases gradually. The magnetic pressure gradient elongates the magnetic flux tube radially. Eventually, the thin current sheet is formed in the anti-parallel magnetic field just above the disk. In this thin current sheet, the magnetic reconnection is caused by the anomalous resistivity and the magnetic energy is released rapidly. This model of a proto-star seems to be directly applicable to the plasma dynamics in the black hole magnetosphere. In the magnetosphere around the rotating black hole, the magnetic bridges between the ergosphere and the disk are twisted by the plasma in the ergosphere mainly because of the frame-dragging effect. This model may explain the correlation between the flare and the knot ejection from M87 and microQSOs.

In section 2, we summarize the results of ideal GRMHD simulations of jet formation in black hole magnetospheres. In section 3, we mention the observation of correlation between the flare and the knot ejection with respect to M87 and introduce a model of magnetic reconnection for the observation. We discuss the equations required by the model beyond the ideal GRMHD. In section 4, we introduce a set of generalized GRMHD equations. In section 5 , we show some peculiar phenomena suggested by the generalized GRMHD equations briefly. In section 6 , we present summary and some discussions.

\section{Ideal GRMHD simulations of jet formation}

In models of black hole magnetospheres, we have to consider the interaction between the plasmas and magnetic field around a black hole. It is well known that the interaction between the plasmas and magnetic field often causes complex phenomena. In such case, numerical simulations provide strong tools to find the solution of the phenomena. The simplest treatment of such interaction is given by GRMHD equations. Recently, numerical simulations of GRMHD had been performed by some groups. Most of them assumed the electric resistivity is zero (ideal GRMHD), while a few simulations of relativistic MHD with resistivity (resistive RMHD) were performed within special relativistic framework (Watanabe \& Yokoyama 2006). Here, we review the results of ideal GRMHD simulations of the jet formation in the black hole magnetospheres.

In the black hole magnetosphere, there may be several types of magnetic field lines. Some of open magnetic field lines cross the disk around the black hole, while some of them cross the black hole. Some of the closed magnetic field lines are anchored to the disk. Here, we focus on the closed magnetic field lines, called "magnetic bridges", between the ergosphere and black hole accretion disk. Plasmas in the ergosphere rotate faster than the local light speed because of the frame-dragging effect around the black hole. Usually, angular velocity of the plasma in the ergosphere is much faster than that of the accretion disk around the black hole. The rotation of the plasma in the ergosphere twists the magnetic bridges between the ergosphere and the disk rapidly when the black hole 
rotates rapidly. The magnetic pressure increases in the magnetic bridges quickly. This rapid increase in the magnetic pressure will brow off the plasma around the ergosphere and will form the relativistic jet (Fig. 1, panels (a) and (b)). It is expected that thin current sheet is formed in the elongated magnetic bridges and a large amount of magnetic energy is stored (Fig. 1 (c)). When the current sheet becomes thin and the current density exceeds a critical value, the magnetic reconnection is caused and a large amount of magnetic energy is released (Fig. $3(\mathrm{~d})$ ). The upward reconnection jet pushes and accelerates a plasmoid and the downward jet may heat up the inner edge of the disk. The ejection of the plasmoid and the heating of the disk edge may correspond to the knot ejection and high energy flare, respectively.

A numerical simulation of this type of jet formation was performed by Koide, Shibata, \& Kudoh (2006). The simulation showed that the magnetic bridges are twisted by the plasma in the ergosphere and the disk. The twist by the plasma in the ergosphere is dominant and the magnetic pressure increases rapidly near the ergosphere. The magnetic

(a)

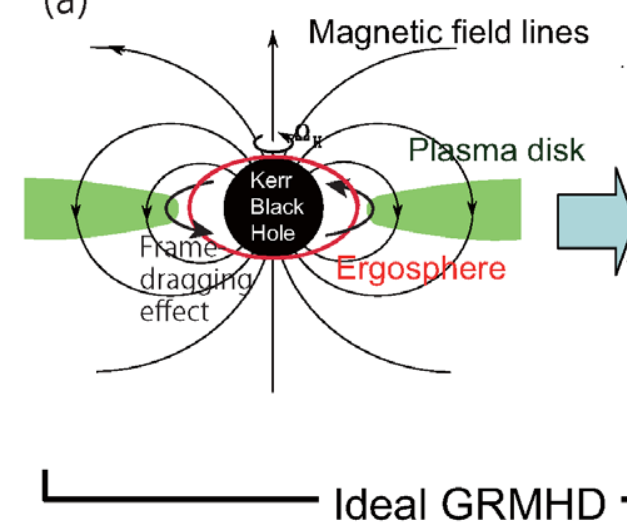

(C)

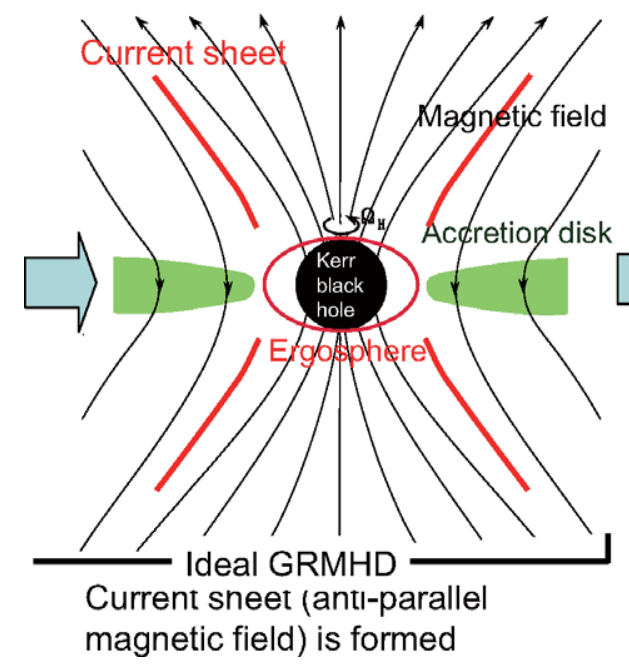

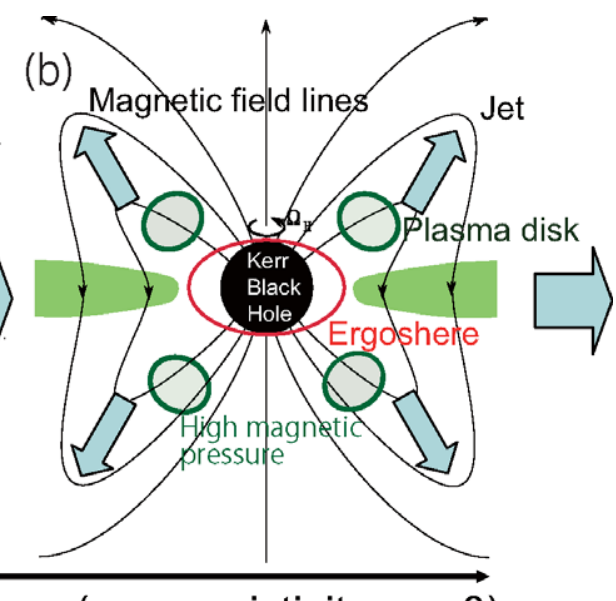

(zero resistivity: $\eta=0$ )

(d)

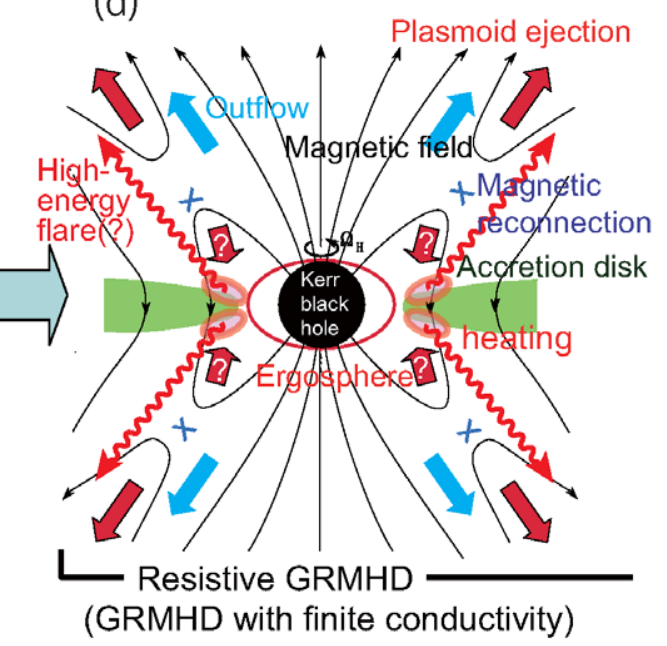

Figure 1. Dynamics of magnetic bridges between the ergosphere and accretion disk around the rapidly rotating black hole. 
pressure gradient brows off the plasma around the ergosphere radially. The radial outflow is collimated by the magnetic tension to become a jet. At $t=110 \tau_{\mathrm{S}}$, the maximum velocity of the jet is $\sim 0.6 c$, sub-relativistic, where $c$ is the light speed in vacuum, $\tau_{\mathrm{S}} \equiv r_{\mathrm{S}} / c$ is the time unit, and $r_{\mathrm{S}}$ is the Schwarzschild radius. In this calculation, we set a strong magnetic field as the initial condition of magnetic field, where the 4-Alfven velocity is $0.6 c$. In such a strong magnetic field case, the small-scale MRI is suppressed by the magnetic tension and then no small-scale turbulence is formed. In this situation, the analysis of the simulation is relatively easy because of the simple structure without turbulence of MRI. This enable us to find the thin current sheet formation clearly at the trough of the magnetic pressure, $B_{\phi}^{2}=0$, above the disk (Figs. 2 and 3). The trough of magnetic field exists along the marginal surface of the magnetic flux surface (Fig. 3 (a)). The magnetic pressure gradient pushes the plasma toward the trough of the magnetic pressure and forms thin current sheet naturally (Fig. 3 (b)). Then, a thin current sheet is formed at the trough of the magnetic pressure as a natural consequence.

Similar thin current sheet formation was also found in the non-relativistic MHD simulation of magnetic bridges between a proto-star and a disk as mentioned in section 1 (Hayashi et al. 1996). They, furthermore, showed the formation of fast ejection of the plasmoid due to the fast magnetic reconnection at the current sheet with anomalous resistivity. This mechanism of magnetic reconnection is applicable around the current sheet near the black hole suggested by the results of the ideal GRMHD simulations. When this magnetic reconnection mechanism occurs around the rapidly rotating black hole, the plasmoid ejection will be observed as a knot-ejection and the heating of inner edge of the disk due to the collision with the downward reconnection flow will be observed as a high energy flare (Fig. 1 (d)). It is noted that if the outflow velocity around the current sheet is larger than the Alfven velocity at the region, the downward reconnection flow can not reach to the inner edge of the disk (this is expressed by question marks in Fig. 1 (d)). In such case, the collision between reconnection outflow and disk plasma can not happen and the heating of the inner edge of the disk is not realized. In this case, the plasmoid is ejected without the heating of the inner disk. In the numerical result of Hayashiet al.(1996), they did not find the heating of the inner edge of the disk. This may be explained by the above process. We also mention that, in such case, the direction of the reconnection outflow is almost the same as that of the bulk plasma outflow driven
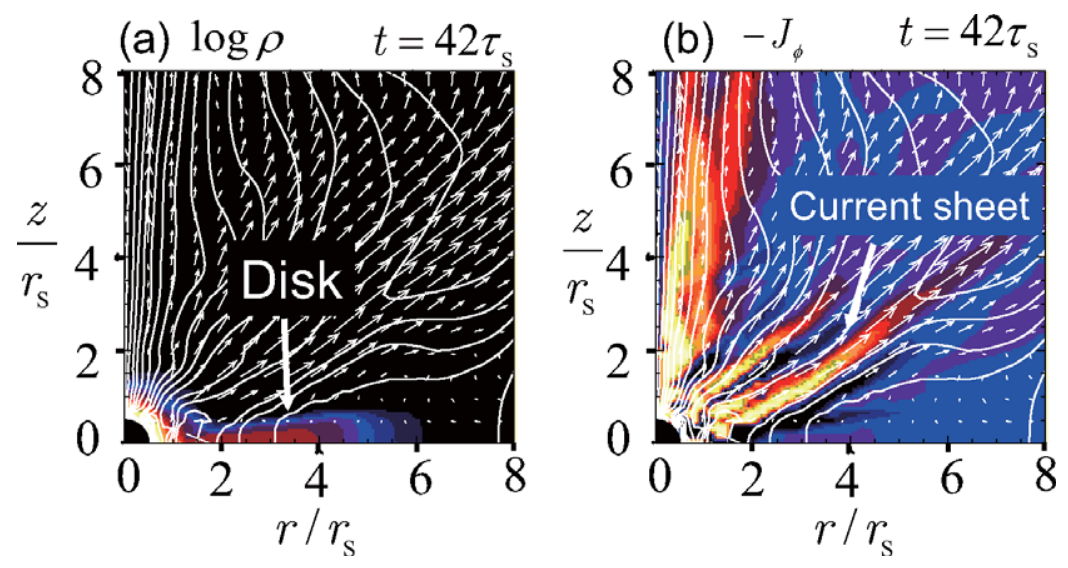

Figure 2. Density $\rho$ and azimuthal component of current density $J_{\phi}$ around the rotating black hole at $t=42 \tau_{\mathrm{S}}$ of the GRMHD simulation by Koide et al. (2006). 
by the magnetic pressure. It is expected that the magnetic reconnection accelerates the magnetically driven outflow faster more than a factor of 2 with 4 -velocity.

On the other hand, a much longer-term, much wider-region simulation of ideal GRMHD was performed by McKinney (2006). The time duration reached to $t=7000 \tau_{\mathrm{S}}$ and the spatial range spreads to the area of $r \leqslant 10^{4} r_{\mathrm{S}}$. The numerical result showed that the jet is accelerated gradually along the jet and its velocity reaches to the Lorentz factor $\Gamma \approx 7$ at $r=1000 r_{\mathrm{S}}$. His numerical simulation also showed that the density $\rho$ and magnetic field $B_{\perp}$ decrease monotonically along the jet as the distance from the black hole. Because intensity of synchrotron radiation is roughly estimated by $P \propto \rho B_{\perp}^{2}$, this suggests the radio image of the jet is monotonically continuous and has no knot within the ideal GRMHD models.

\section{A model beyond ideal GRMHD}

Multi-wavelength observations of M87 with radio, X-ray, and $\gamma$-ray showed the correlation between the high energy ( $\gamma$-ray) flare of the nucleus (accretion disk around the central black hole) and the knot ejection from the nucleus (Acciari et al. 2009). The knot ejection was observed by the subtraction of the average image over 11 period between January and August 2007 from the image of one period on 5 May 2008 of VLBA. No knot was observed in the subtraction from the image on 25 February 2008 just before the high energy flare. The former subtracted image also shows that the radio emission from the radio core is dominant during the radio flare. The core is identified to the M87 nucleus because of no spatial shift within $6 r_{\mathrm{S}}$ during the radio flare. Similar correlation between a high energy flare and a radio knot ejection was observed in micro-QSOs more frequently (Mirabel \& Rodriguez 1998). These drastically time-varying high energy phenomena correlating the flare and the knot ejection may be explained by the magnetic reconnection model mentioned in Sect. 2 .

To investigate the magnetic reconnection model in the black hole magnetosphere, the simplest approximation of the plasma is given by GRMHD equations with finite resistivity, called "standard resistive GRMHD". However, the standard resistive GRMHD has a problem with respect to causality. The problem comes from the "standard" relativistic Ohm's law, $U^{\nu} F^{\mu}{ }_{\nu}=\eta\left[J^{\mu}-\left(U^{\nu} J_{\nu}\right) U^{\mu}\right]$, where $U^{\mu}$ is the 4-velocity, $J^{\mu}$ is the 4-current density, $F_{\mu \nu}$ is the electromagnetic field tensor, and $\eta$ is the resistivity. Here, the electric
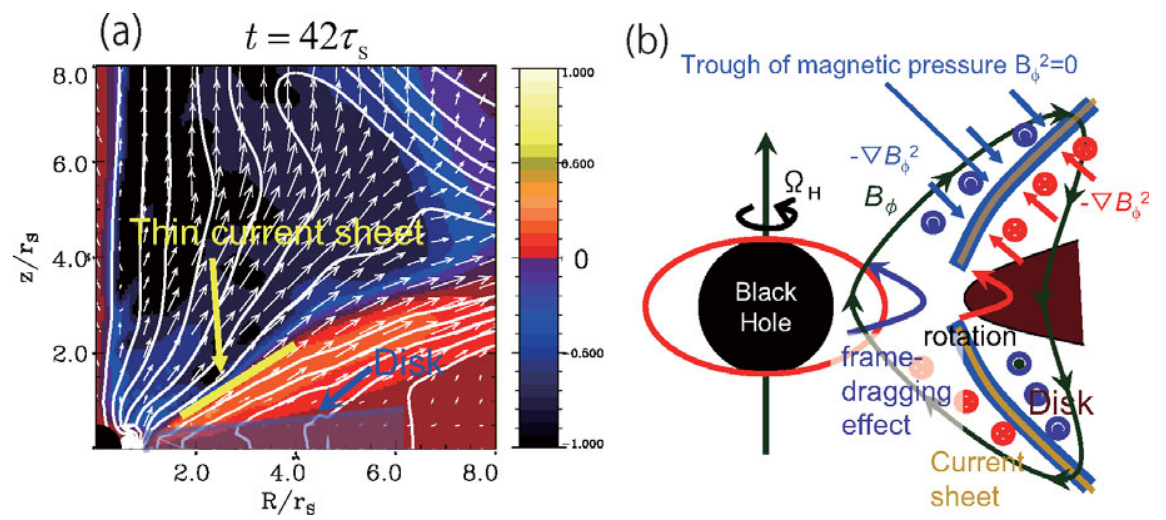

Figure 3. Azimuthal component of magnetic field $B_{\phi}$ and the location of current sheet (thick solid line) at $t=42 \tau_{\mathrm{S}}$ of the GRMHD simulation by Koide et al. (2006) (a) and the formation mechanism of the thin current sheet (b). 
field is given by $E_{i}=F_{i 0}$ and the magnetic field is $F_{i j}=\sum_{k} \epsilon_{i j k} B_{k}\left(\epsilon_{i j k}\right.$ is the LeviCivita symbol), where the alphabetic index $(i, j, k)$ runs from 1 to 3 . Hereafter, we use the unit system where light speed is unity and the energy densities of electric field $\boldsymbol{E}$ and magnetic field $\boldsymbol{B}$ are given by $E^{2} / 2$ and $B^{2} / 2$ in the Minkowski space-time, respectively.

In the ideal GRMHD case, the electromagnetic wave never propagates. On the other hand, in the resistive GRMHD case, the electromagnetic wave can propagate. The dispersion relation of the wave in uniform, rest plasma is $\omega^{2}+\frac{i}{\eta} \omega-k^{2}=0$, where $\omega$ and $k$ are the complex frequency and the wave number, respectively. The group velocity of the wave, $v_{\mathrm{g}}=\frac{\partial \omega}{\partial k}=\frac{k}{\sqrt{k^{2}-(2 \eta)^{-2}}}>1$, is always greater than the light speed. This means the breakdown of causality. To solve this problem, we reconsider the GRMHD equations based on the general relativistic two-fluid equations (Koide 2010a).

\section{Generalized GRMHD equations}

We derive generalized GRMHD equations based on general relativistic two-fluid equations. For simplicity, we assumed that the plasma is composed of two fluids, where one fluid consists of positively charged particles with mass $m_{+}$and electric charge $e$, and the other fluid consists of negatively charged particles with mass $m_{-}$and charge $-e$. The relativistic equations of the two fluids and the Maxwell equations are

$$
\begin{aligned}
\nabla_{\nu}\left(n_{ \pm} U_{ \pm}^{\nu}\right) & =0, \\
\nabla_{\nu}\left(h_{ \pm} U_{ \pm}^{\mu} U_{ \pm}^{\nu}\right) & =-\nabla^{\mu} p_{ \pm} \pm e n_{ \pm} g^{\mu \sigma} U_{ \pm}^{\nu} F_{\sigma \nu} \pm R^{\mu}, \\
\nabla_{\nu}{ }^{*} F^{\mu \nu} & =0, \quad \nabla_{\nu} F^{\mu \nu}=J^{\mu},
\end{aligned}
$$

where variables with subscripts, plus $(+)$ and minus $(-)$, are those of the fluid of positively charged particles and of the fluid of negative particles, respectively. Here, $n_{ \pm}$is the proper particle number density, $p_{ \pm}$is the proper pressure, $h_{ \pm}$is the relativistic enthalpy density, $\nabla_{\mu}$ is the covariant derivative, ${ }^{*} F^{\mu \nu}$ is the dual tensor of $F_{\mu \nu}$, and $R^{\mu}$ is the frictional 4 -force density between the two fluids.

To derive one-fluid equations of the plasma, we define the average and difference variables as follows:

$$
\begin{aligned}
\rho & =m_{+} n_{+} \gamma_{+}^{\prime}+m_{-} n_{-} \gamma_{-}^{\prime}, \quad n=\frac{\rho}{m}, \\
p & =p_{+}+p_{-}, \quad \Delta p=p_{+}-p_{-}, \\
U^{\mu} & =\frac{1}{\rho}\left(m_{+} n_{+} U_{+}^{\mu}+m_{-} n_{-} U_{-}^{\mu}\right), \quad J^{\mu}=e\left(n_{+} U_{+}^{\mu}-n_{-} U_{-}^{\mu}\right), \\
h & =n^{2}\left(\frac{h_{+}}{n_{+}^{2}}+\frac{h_{-}}{n_{-}^{2}}\right), \quad \Delta h=\frac{m n^{2}}{2}\left(\frac{h_{+}}{m_{+} n_{+}^{2}}-\frac{h_{-}}{m_{-} n_{-}^{2}}\right), \\
h^{\ddagger} & =h-\Delta \mu \Delta h, \quad \Delta h^{\sharp}=\Delta \mu h-\frac{1-3 \mu}{2 \mu} \Delta h,
\end{aligned}
$$

where $\gamma_{ \pm}^{\prime}$ is the Lorentz factor of the two fluids observed by the local center-of-mass frame of the plasma and $m=m_{+}+m_{-}$is regarded as the typical mass of the plasma particle. Hereafter, a prime is used to denote the variables of the center-of-mass frame of the plasma. Using the above variables, the normalized reduced mass, $\mu=m_{+} m_{-} / m^{2}$, and the normalized mass difference of the positively and negatively charged particles, $\Delta \mu=\left(m_{+}-m_{-}\right) / m$, we obtain

$$
\begin{aligned}
\nabla_{\nu}\left(\rho U^{\nu}\right) & =0, \\
\nabla_{\nu} T^{\mu \nu} & =0,
\end{aligned}
$$




$$
\frac{1}{n e} \nabla_{\nu}\left(\frac{\mu h}{n e} Q^{\mu \nu}\right)=\frac{1}{2 n e} \nabla^{\mu}(\Delta \mu p-\Delta p)+\left(U^{\nu}-\frac{\Delta \mu}{n e} J^{\nu}\right) F_{\nu}^{\mu}-\eta\left[J^{\mu}-\rho_{\mathrm{e}}^{\prime}(1+\Theta) U^{\mu}\right],
$$

where $\rho_{\mathrm{e}}^{\prime}=-J^{\nu} J_{\nu}$ is the charge density observed by the plasma local rest frame, and the energy-momentum tensor $T^{\mu \nu}$ and "charge-current density tensor" $Q^{\mu \nu}$ are given by

$$
\begin{aligned}
T^{\mu \nu} & \equiv p g^{\mu \nu}+h U^{\mu} U^{\nu}+\frac{\mu h^{\ddagger}}{(n e)^{2}} J^{\mu} J^{\nu}+\frac{2 \mu \Delta h}{n e}\left(U^{\mu} J^{\nu}+J^{\mu} U^{\nu}\right)+F_{\sigma}^{\mu} F^{\mu \sigma}-\frac{1}{4} g^{\mu \nu} F^{\kappa \lambda} F_{\kappa \lambda}, \\
Q^{\mu \nu} & \equiv \frac{e n}{h}\left[\frac{h^{\ddagger}}{n e}\left(U^{\mu} J^{\nu}+J^{\mu} U^{\nu}\right)+2 \Delta h U^{\mu} U^{\nu}-\frac{\Delta h^{\sharp}}{(n e)^{2}} J^{\mu} J^{\nu}\right] .
\end{aligned}
$$

Here, we used the relation, $R^{\mu}=-\eta n e\left[J^{\mu}-\rho_{\mathrm{e}}^{\prime}(1+\Theta) U^{\mu}\right]$, where $\Theta$ is the rate of equipartition with respect to the thermalized energy due to friction (see Appendix A of Koide (2009)).

These equations show the inertia effect and momentum of the electric charge and current (3rd and 4th terms of the right-hand side of Equation (4.12) and left-hand side of Equation (4.11)), and thermo-electromotive force (1st term of right-hand side of Equation (4.11)), and Hall effect (2nd term in bracket of factor of 2nd term of right-hand side of Equation (4.11)). When we neglect these terms, there equations reduce to the standard resistive GRMHD equations. Then, we call these equations "generalized GRMHD equations". It is noted that if

$$
\eta<(2.5 \sim 3) \frac{m}{e \rho} \sqrt{\mu h^{\dagger}},
$$

we can prove that causality is kept (Koide 2009). The condition (4.14) is identical to the definition of plasma, that is, the plasma parameter is much larger than unity, where we used the Spitzer resistivity (Bellan 2006).

Now, we found the basic equations for the resistive GRMHD calculation within causality (Koide 2010a). These equations are much complicated compared to the standard resistive GRMHD, thus the calculations with these equations are more difficult.

\section{Peculiar phenomena suggested by generalized GRMHD equations}

We mention two peculiar phenomena suggested by the generalized GRMHD equations. When we write the generalized Ohm's law among the generalized GRMHD equations using $3+1$ formalism, we find new types of electromotive forces due to gravitation, shear of frame-dragging, and centrifugal force of the current. With respect to the gravitational electromotive force $\boldsymbol{E}_{\text {grv }}$, we obtain a formula, $\boldsymbol{E}_{\text {grv }}=\frac{2 \mu h^{\ddagger}}{(n e)^{2} \gamma} \nabla(\ln \alpha) \rho_{\mathrm{e}}^{\dagger}$, where $\alpha$ is the lapse function and $\rho_{\mathrm{e}}^{\dagger}=Q^{00}$ is a kind of electric density (Koide 2010a). Then, if charge separation occurs at the current sheet between the azimuthal anti-parallel magnetic field near the black hole, the gravitational electromotive force may cause the magnetic reconnection without the resistivity, while frame dragging and centrifugal electromotive forces never change the topology of the magnetic field configuration.

We also mention a result of linear analysis of charge separation in the disk plasma rotating circularly around the rotating black hole. It shows that the charge separation is stable outside of the inner most stable orbit (ISCO) and then plasma oscillation is found. On the other hand, inside of the ISCO, the charge separation becomes unstable when the plasma frequency is small enough (Koide 2010b). The charge separation induced by this instability may cause a magnetic reconnection due to the gravitational electromotive 
force without resistivity. The inflow of the charged disk due to the instability into the black hole may charge up the black hole.

\section{Summary and dreams in future}

After the brief review of the results of ideal GRMHD simulations, we showed a model beyond the ideal GRMHD. Especially, we focus on the resistive GRMHD to simulate the magnetic reconnection in the black hole magnetosphere. This magnetic reconnection model comes from the modified model of a solar flare, and it can explain the correlation between the high energy flares and the radio knot ejections of M87 and micro-QSOs (Acciari et al. 2009; Mirabel \& Rodrguez 1998). With respect to the basic equations beyond the ideal GRMHD within causality, we introduced the generalized GRMHD equations. We showed two peculiar phenomena suggested by these equations. To investigate the plasma dynamics in the black hole magnetosphere using the generalized GRMHD equations, we have to use new numerical calculation technique of these equations. The equations of continuity and energy and momentum conservation are similar to those of the ideal GRMHD equations. However, the generalized Ohm's law is drastically different from the Ohm's law of the standard resistive GRMHD equations. In the generalized Ohm's law, we have to consider the time derivative of current density, which is neglected in the standard GRMHD. This term is important to keep causality. Then, we have to develop entirely new numerical method for it. Unfortunately, we have no idea of the numerical calculation yet. This is our important, urgent task.

Once we develop the numerical code of the generalized GRMHD with a high magnetic Reynolds number, we could test the magnetic reconnection model of the flare-knot ejection correlation phenomenon around the black holes. In the calculation, we have to set the magnetic Reynolds number so large that a large amount of magnetic energy in the elongated magnetic bridges is stored, while the resistivity must exceed the numerical resistivity. It is noted that the numerical resistivity is not negligible in the recent ideal GRMHD simulations so that thin current sheet was diffused to reduce the magnetic energy stored in the magnetic bridges artificially. In such case, explosive phenomena expected in the very high magnetic Reynolds number case are suppressed. To calculate an explosive phenomena, we have to perform numerical simulations with very small numerical resistivity before we perform the resistive GRMHD calculation with large magnetic Reynolds number. Numerical simulations of peculiar phenomena suggested by the generalized GRMHD equations are also interesting.

\section{Acknowledgements}

We thank Mika Koide for her helpful comments on this manuscript. This work was supported in part by the Science Research Fund of the Japanese Ministry of Education, Culture, Sports, Science, and Technology.

\section{References}

Acciari, V. A. et al. 2009, Science, 325, 444.

Bellan, P. M. 2006, Fundamentals of Plasma Physics (Cambridge Univ., Cambridge).

Hayashi, M. R., Shibata, K., \& Matsumoto, R. 1996, ApJ, 468, L37.

Koide, S. 2009, ApJ, 696, 2220.

Koide, S. 2010a, ApJ, 708, 1459.

Koide, S. 2010b, arXiv:1009.6041.

Koide, S., Shibata, K., \& Kudoh, T. 2006, Phys. Rev. D, 74, 044005. 
McKinney, J. C. 2006, Mon. Not. R. Acad. Soc., 368, 1561.

Mirabel, I. F. \& Rodriguez, L. F. 1998, Nature, 392, 673.

Shibata, K., Masuda, S., Shimojo, M., Hara, H., Yokoyama, T., Tsuneta, S., Kosugi, T., Ogawara, Y. 1995, ApJ, 451, L83.

Watanabe, N. \& Yokoyama, T. 2006, ApJ, 647, L123.

\section{Discussion}

KIRK: I agree that two-fluid generalization of GRMHD is very important. In particular, the charge-separation instability you described is potentially crucial. Do you expect that this or other instabilities will be strongly affected by non-axisymmetric perturbations?

KoIDE: That is an important question. However, I did not perform a linear analysis with the non-axisymmetric perturbation. I would like to perform the linear analysis in the future.

YUAN: Can you comment on the existence of the "magnetic bridge" initial configuration?

KoIDE: During accretion, the inner accretion flow loses angular momentum, plunging into the $\mathrm{BH}$, and resulting in the formation of this configuration.

NisHIKAWA: The idea of charge-separation instability may be a mechanism of jet formation seen in GRPIC simulations in Watson \& Wikshikawa (2010)?

KoIDE: I think the charge-separation instability causes a current parallel to the equatorial plane only. Then, it does not drive a jet nor an outflow.

De Gouveia Dal Pino: In order to have efficient reconnection events if you inject weak turbulence in the reconnection site, this eventually speeds up reconnection making it very fast even with small levels of resistivity as suggested by Vishniac \& Lazarian (1999) and recently tested by 3D numerical MHD simulations by Kowal et al. (2009).

KoIDE: That is interesting. That mechanism would realize the anomalous resistivity in the current sheets formed in black hole magnetospheres. 